\title{
TIR-domain-containing adapter-inducing interferon- $\beta$ (TRIF) regulates Th17-mediated intestinal immunopathology in colitis
}

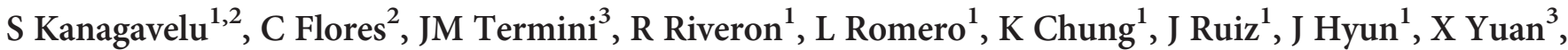 \\ $\mathrm{J} \mathrm{Dagvadorj}^{4}$ and M Fukata ${ }^{1,2,5}$
}

Gastrointestinal mucosa reserves abundant Th17 cells where host response to commensal bacteria maintains Th17-cell generation. Although functional heterogeneity and dynamic plasticity of Th17 cells appear to be involved in chronic inflammatory disorders, how their plasticity is regulated in intestinal mucosa is unknown. Here we show that innate TRIF signaling regulates intestinal Th17-cell generation and plasticity during colitis. Absence of TRIF in mice resulted in increased severity of experimental colitis, which was associated with aberrant generation of Th17 cells especially of interferon (IFN)- $\gamma$-expressing Th17 cells in the lamina propria. The abnormal generation and plasticity of Th17 cells involved impaired expression of interleukin (IL)-27p28 by lamina propria macrophages but not dendritic cells. Treatment of TRIF-deficient mice with IL-27p28 during colitis reduced the number and IFN- $\gamma$ expression of Th17 cells in the intestine. In vitro, TRIF-deficient macrophages induced more Th17 cells than wild-type (WT) macrophages during coculture with WT naive T cells in response to cecal bacterial antigen. Many of Th17 cells induced by TRIF-deficient macrophages expressed IFN- $\gamma$ due to impaired expression of IL-27p28 by macrophages and defective activation of STAT1 in T cells. These results outline TRIF-dependent regulatory mechanism by which host response to intestinal bacteria maintains Th17-cell-mediated pathology during colitis.

\section{INTRODUCTION}

An aberrant mucosal T-cell response to intestinal commensal bacteria has been implicated in the pathogenesis of inflammatory bowel disease (IBD). Since the discovery of T-helper (Th)17 cells, multiple studies have shown a massive infiltration of Th17 cells in IBD mucosa, highlighting a role for Th17 cells in IBD pathogenesis. ${ }^{1,2}$ Genetic studies have identified strong associations between gene mutations in interleukin-23 receptor $(I L-23 R)$ and the IL-17 receptor E-like (IL-17REL) with the development of IBD, further supporting an important link between Th17 cells and IBD. ${ }^{3}$ On the basis of these findings, therapeutics designed to alter the IL-23-IL-17 axis have been developed. However, clinical trials produced disappointing results, ${ }^{4-6}$ suggesting that further research is needed to translate the intestinal Th17-cell phenotype to therapeutic advancements in IBD.

Accumulating evidence has suggested that intestinal Th17 cells consist of immunopathogenic and regulatory subpopulations. ${ }^{7-9}$ It has been shown that immunopathogenic Th17 cells have a strong effector phenotype but that regulatory Th17 cells are capable of reducing inflammation. ${ }^{10,11}$ In addition, recent reports have shown that Th17 cells can undergo transformation into other Th-cell subsets. ${ }^{12}$ IL- $17^{+}$Foxp ${ }^{+}$cells as well as IL- $17^{+}$interferon (IFN) $\gamma^{+}$T cells have been identified in lamina propria of patients with Crohn's disease, suggesting an involvement of Th17-cell plasticity in the pathogenesis of

\footnotetext{
${ }^{1}$ Division of Gastroenterology, Department of Medicine, University of Miami Miller School of Medicine, Miami, Florida, USA. ${ }^{2}$ Division of Gastroenterology, Department of Medicine, F. Widjaja Foundation, Inflammatory Bowel and Immunology Research Institute, Cedars-Sinai Medical Center, Los Angeles, California, USA. ${ }^{3}$ Department of Microbiology and Immunology, University of Miami Miller School of Medicine, Miami, Florida, USA. ${ }^{4}$ Division of Infectious Diseases and Immunology, Department of Biomedical Science, Medicine and Pediatrics, Cedars-Sinai Medical Center, Los Angeles, California, USA and ${ }^{5}$ Department of Cell Biology, University of Miami Miller School of Medicine, Miami, Florida, USA. Correspondence: M Fukata, (Masayuki.fukata@cshs.org)
} 
Crohn's disease. ${ }^{13,14}$ However, the precise mechanism regulating the dynamic state of Th17-cell plasticity remains to be determined.

The absence of Th17 cells in the lamina propria of germ-free mice suggests that commensal bacteria have a role in intestinal Th17-cell differentiation. ${ }^{15,16}$ Toll-like receptors (TLRs) are a family of pattern-recognition receptors that induce cytokines and chemokines by recognizing microorganisms. Although most key cytokines that direct Th17-cell differentiation are known to be induced by TLR signaling in response to bacteria, little is known about how it is regulated during intestinal inflammation. Mice that are deficient in the two major adapter molecules of TLRs, MyD88 and TRIF, have similar numbers of Th17 cells in the lamina propria compared with wild-type (WT) mice, ${ }^{15}$ whereas mice that are deficient in only MyD88 have significantly reduced intestinal Th17 cells. ${ }^{17}$ Thus, the normal generation of intestinal Th17 cells in MyD88/TRIFdouble-deficient mice could be explained if MyD88 and TRIF signaling positively and negatively regulate mucosal Th17-cell generation, respectively. In fact, TRIF-deficient mice demonstrated increased Th17-cell generation than WT mice in experimental autoimmune encephalomyelitis. ${ }^{18}$ In addition, TRIF-deficient dendritic cells (DCs) have been shown to shift Tregs to Th17 cells in vivo. ${ }^{19}$ These findings suggest an important regulatory role of TRIF signaling in intestinal inflammation especially in the context of Th17-cell-mediated inflamatory responses against commensal bacteria.

In this study, we examined the role of TRIF signaling in Th17-cell generation during intestinal inflammation using the 2,4,6-trinitrobenzenesulphonic acid (TNBS) colitis model. We found that TRIF regulates Th17-cell plasticity with Th1 cells, but not with Tregs, in the setting of intestinal inflammation. The underlying mechanism of impaired regulation of Th17/ Th1 plasticity in Trif ${ }^{\mathrm{LPS} 2}$ mice involves defective induction of IL-27p28 from lamina propria macrophages. TRIF-mediated induction of IFN- $\beta$ and IL-27p28 in macrophages led to phosphorylation of the transcription factor STAT1 in Th17 cells during their differentiation, which regulated the IFN- $\gamma$ expression. Our results provide an important insight into the potent regulatory mechanism of Th17-cell pathology in the subset of patients with IBD defined by IFN- $\gamma$-expressing Th17 cells in the intestine.

\section{RESULTS}

Trif $^{\text {LPS2 }}$ mice manifest more severe colitis than WT mice In order to examine the role of TRIF signaling in T-cellmediated intestinal inflammation, we induced TNBS colitis in WT and Trif ${ }^{\mathrm{LPS} 2}$ mice. In the course of TNBS colitis, Trif ${ }^{\mathrm{LPS} 2}$ mice lost more body weight than WT mice (Figure 1a). Colonoscopic examination at day 14 demonstrated larger ulcerations with exudate and paler and edematous mucosa in Trif ${ }^{\mathrm{LPS} 2}$ mice compared with WT mice (Figure 1b). Macroscopically, colons of Trif ${ }^{\mathrm{LPS} 2}$ mice were shorter and their weight to length ratio was significantly greater than WT colons (Figure 1c). The histological severity of colitis was also signi- ficantly greater in Trif ${ }^{\mathrm{LPS} 2}$ mice than WT mice (Figure 1d). Although total cell numbers in the mesenteric lymph node (MLN) were similar between WT mice and Trif ${ }^{\mathrm{LPS} 2}$ mice, the proportion of $\mathrm{CD} 69^{+}$-activated lymphocytes was greater in Trif $^{\mathrm{LPS} 2}$ mice than WT mice (Figures 1e and $\mathbf{f}$ ). These results indicate that absence of TRIF signaling promotes more severe intestinal inflammation associated with greater immune activation in TNBS colitis.

\section{Absence of TRIF induces greater expansion of lamina propria Th17 cells during colitis}

To determine the mechanism underlying the increased severity of colitis in Trif ${ }^{\mathrm{LPS} 2}$ mice, we measured the expression of several key cytokines in the colon that have been implicated in severity of colitis. Although mucosal expression of IFN- $\gamma$, IL-12p35, tumor necrosis factor- $\alpha$ messenger RNA was similar between TNBS-treated WT and Trif ${ }^{\mathrm{LPS} 2}$ mice, the expression of IL-17 in the colon was significantly greater in Trif ${ }^{\mathrm{LPS} 2}$ mice compared with WT mice after induction of TNBS colitis (Figure 2a). Consistent with the gene expression data, the increased IL-17 protein production in ex vivo colon culture was confirmed in Trif $^{\mathrm{LPS2}}$ mice compared with WT mice (Figure $2 \mathbf{b}$ ). The baseline IL-17 protein production in ex vivo colon culture was similar between Trif $^{\mathrm{LPS} 2}$ mice and WT mice (Figure 2b). Flow cytometry (FCM) analysis demonstrated that the proportion of Th17 cells was significantly higher in Trif ${ }^{\mathrm{LP} 2}$ mice than WT mice (Figure 2c). Trif ${ }^{\mathrm{LPS} 2}$ mice also had more IFN- $\gamma$ expressing lamina propria Th1 cells compared with WT mice, but the difference did not reach statistical significance (Figure 2c). Consistently, the proportion of Th17 cells in the MLN was higher in Trif ${ }^{\mathrm{LPS} 2}$ mice than WT mice, whereas Th1 cells in the MLN were similar between them (Figure 2d). These results indicate that Trif ${ }^{\mathrm{LP} 2}$ mice generate more Th17 cells than WT mice during colitis.

\section{Trif $^{\mathrm{LPS} 2}$ mice have IFN- $\gamma$-expressing Th17 cells during colitis}

Recent reports have shown that Th17 cells can undergo transformation into other Th-cell subsets. ${ }^{12} \mathrm{IFN}-\gamma^{+} \mathrm{IL}-17^{+} \mathrm{T}$ cells have been identified in inflamed lamina propria of human and a mouse model of IBD. ${ }^{13,14,20}$ Given the increased generation of intestinal Th17 cells in Trif ${ }^{\mathrm{LPS} 2}$ mice, we examined whether these Th17 cells also expressed IFN- $\gamma$. FCM showed that almost one-third of IL-17-expressing $\mathrm{CD}^{+}{ }^{+} \mathrm{T}$ cells in the lamina propria and the MLN in Trif ${ }^{\mathrm{LPS} 2}$ mice expressed IFN- $\gamma$, whereas such IFN- $\gamma$-expressing Th17 cells were rare in WT mice (Figure 2e). Neither the increase in Th17 cells nor IFN- $\gamma$ expressing Th17 cells were observed in Trif ${ }^{\mathrm{LS} 2}$ mice prior to TNBS colitis (Supplementary Figure S1 online). On the other hand, severity of colitis has been associated with the abundance and function of regulatory $\mathrm{T}$ cells in the lamina propria. The number of Foxp $3^{+}$Tregs in the lamina propria was similar between WT and Trif ${ }^{\mathrm{LPS} 2}$ mice during TNBS colitis $(6.3 \pm 1.4 \%$ vs. $8.5 \pm 0.6 \%$, respectively). In addition, the cell population that expresses Foxp3 among lamina propria Th17 cells was found in very low numbers in both WT as well as Trif ${ }^{\mathrm{LPS} 2}$ mice (Figure 2f). These results suggest that TRIF signaling regulates 
a

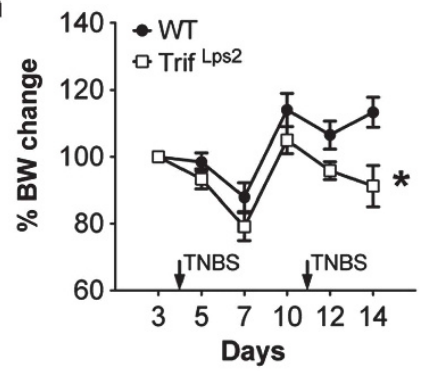

b
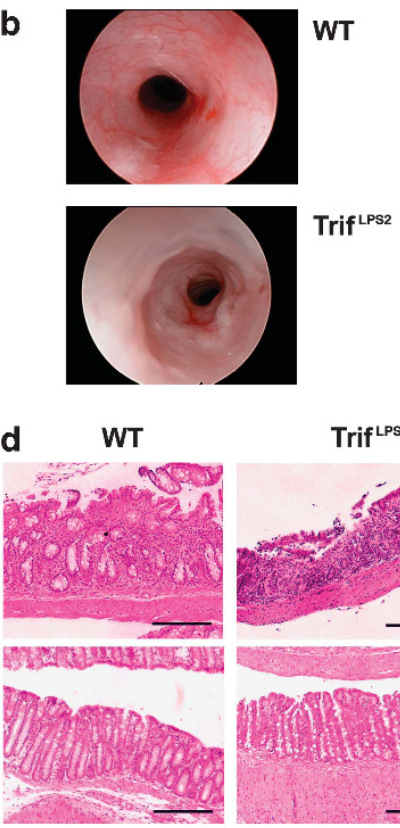
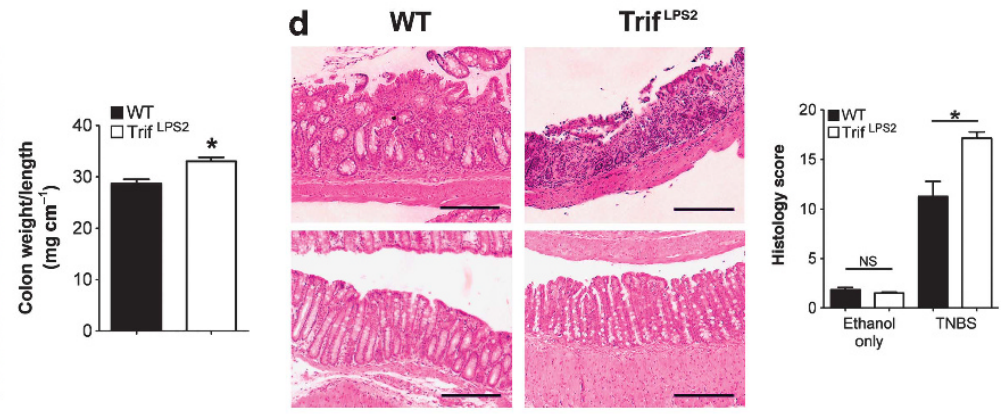

e
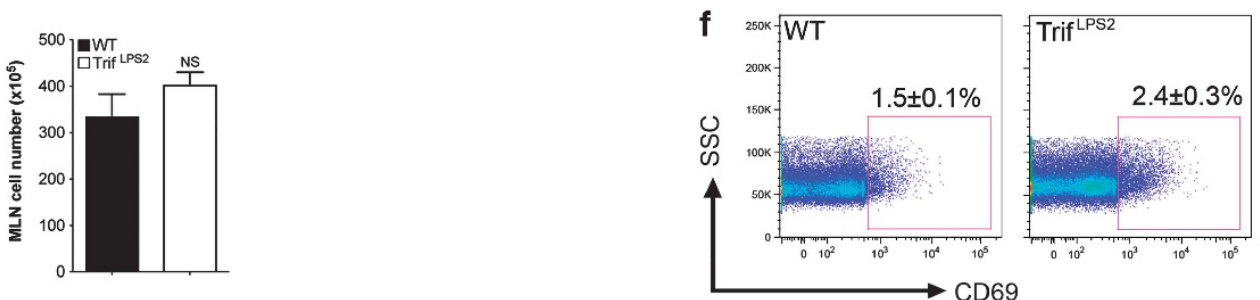

Figure 1 TRIF signaling is protective in 2,4,6-trinitrobenzenesulphonic acid (TNBS)-induced intestinal inflammation. (a) Weight loss in wild-type (WT) and Trif ${ }^{\text {Lps2 }}$ mice during TNBS colitis $\left(n=9\right.$ each, $\left.{ }^{\star} P<0.05\right)$. Combined data from two independent experiments, error bars, s.e.m. (b) Representative endoscopic pictures of colonic mucosa. Nine mice from each genotype underwent colonoscopic examination on day 14. (c) Macroscopic observation of the colon (day 14). The graph shows weight/length ratio of the colon measured after washing out luminal contents and excluding excess liquid ( $n=9$ each, ${ }^{\star} P<0.05$, error bars, s.e.m.). (d) Representative hematoxylin and eosin pictures of TNBS colitis ( $n=9$ each, A and B) and controls ( $n=3$ each, C and D). Original magnification, $\times 20$, Bar $=100 \mu \mathrm{m}$. The graph shows histological scoring for severity of colitis. ${ }^{41}$ Calculated from two independent experiments ( $n=9$ each, highest score of 24 , error bars, s.e.m., $\left.{ }^{\star} P<0.05\right)$. (e) Total cell number in the mesenteric lymph node (MLN) isolated from WT and Trif ${ }^{\llcorner p s} 2$ mice on day 14 ( $n=9$ each, error bars, s.e.m., NS, not significant). (f) Single-cell suspension (SSC) of the MLN was analyzed for CD69 expression by flow cytometry. The number shows mean \pm s.e.m. $(n=4, P<0.05)$. BW, body weight.

intestinal Th17/Th1 plasticity but not Th17/Treg plasticity during intestinal inflammation.

\section{Lamina propria macrophages, but not DCs, from Trif ${ }^{\text {LPS2 }}$ mice skew Th-cell differentiation toward Th17 cells in response to commensal bacteria}

To determine whether the strong Th17-cell differentiation in Trif $^{\mathrm{LPS} 2}$ mice was due to altered response of antigen-presenting cells to commensal bacteria, $\mathrm{CD} 11 \mathrm{c}^{+} \mathrm{F} 4 / 80^{-}$lamina propria DCs (LPDCs) and CD11c ${ }^{-} \mathrm{F} 4 / 80^{+}$macropahges were separately isolated from $\mathrm{WT}$ and Trif ${ }^{\mathrm{LS} 2}$ mice and co-cultured with WT splenic naive $\mathrm{T}$ cells in the presence of cecal bacterial antigen (CBA) $\left(100 \mu \mathrm{g} \mathrm{ml}^{-1}\right)$. Although there was no difference in the rate of Th17 cells generated during 3 days co-culture of LPDCs and naive T cells, Trif ${ }^{\mathrm{LPS} 2} \mathrm{CD} 11 \mathrm{c}^{-} \mathrm{F} 4 / 80^{+}$macrophages generated more Th17 cells than WT macrophages (Figure 3a,c). Likewise, Th1-cell generation was similar in co-cultures with WT LPDCs and Trif ${ }^{\mathrm{LPS} 2}$ LPDCs, but slightly more in co-cultures with $\mathrm{Trif}^{\mathrm{LPS} 2} \mathrm{CD} 11 \mathrm{c}^{-} \mathrm{F} 4 / 80^{+}$ macrophages compared with WT CD11c ${ }^{-} \mathrm{F} 4 / 80^{+}$macrophages (Figure $\mathbf{3 b}, \mathbf{d}$ ). These results indicate that TRIF deficiency in lamina propria macrophages, but not DCs, are prone to generate Th17 cells in response to commensal bacteria.

\section{IL-27p28 expression in lamina propria macrophages is impaired in Trif ${ }^{\text {LPS2 }}$ mice during colitis}

Antigen-presenting cells direct Th-cell differentiation by expressing unique sets of cytokines in response to antigens. We therefore examined the expression of mucosal cytokines associated with Th17-cell differentiation during TNBS colitis (Figure 4a). Real-time PCR of the colon demonstrated similar expression of IL-23p19, IL-6, and TGF- $\beta$ between WT and Trif $^{\mathrm{LPS} 2}$ mice. However, colonic expression of IL-27p28 and IFN- $\beta$ in Trif ${ }^{\mathrm{LPS} 2}$ mice was significantly lower than WT mice. Colonic expression of the other subunit of IL-27 (EBI3) was similar between WT and Trif ${ }^{\mathrm{LPS} 2}$ mice. The altered production of IL-27p28 was confirmed at the protein level using ex vivo colon culture supernatants (Figure 4b). Although WT colon 

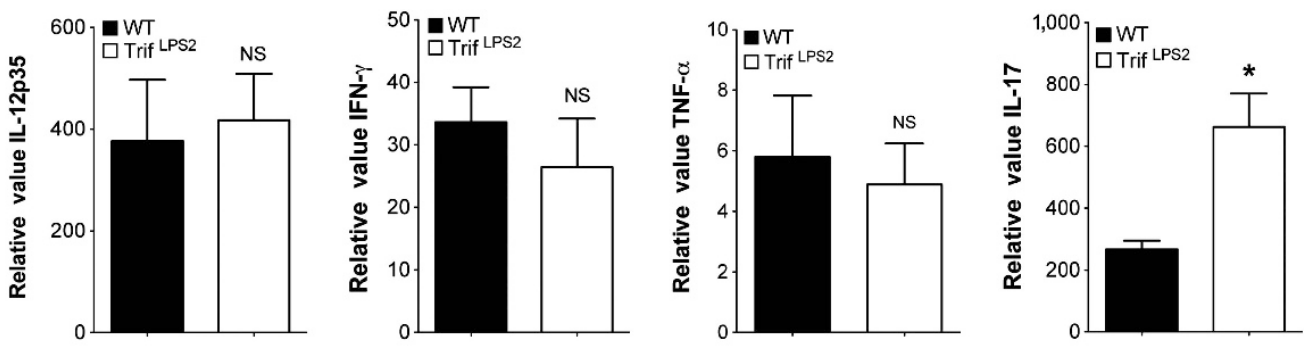

b

C
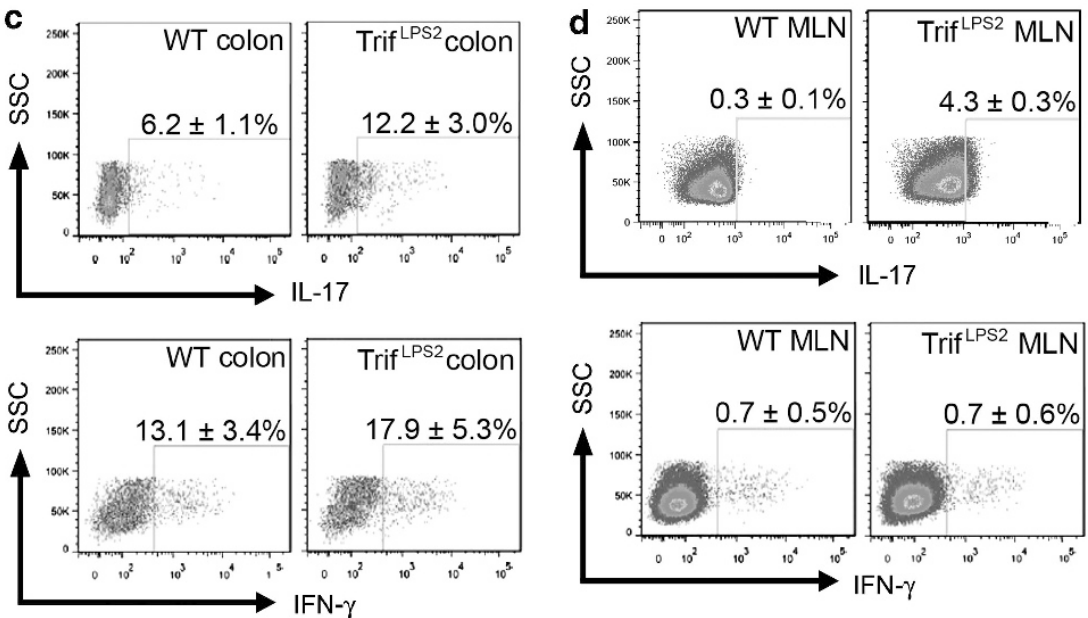

e
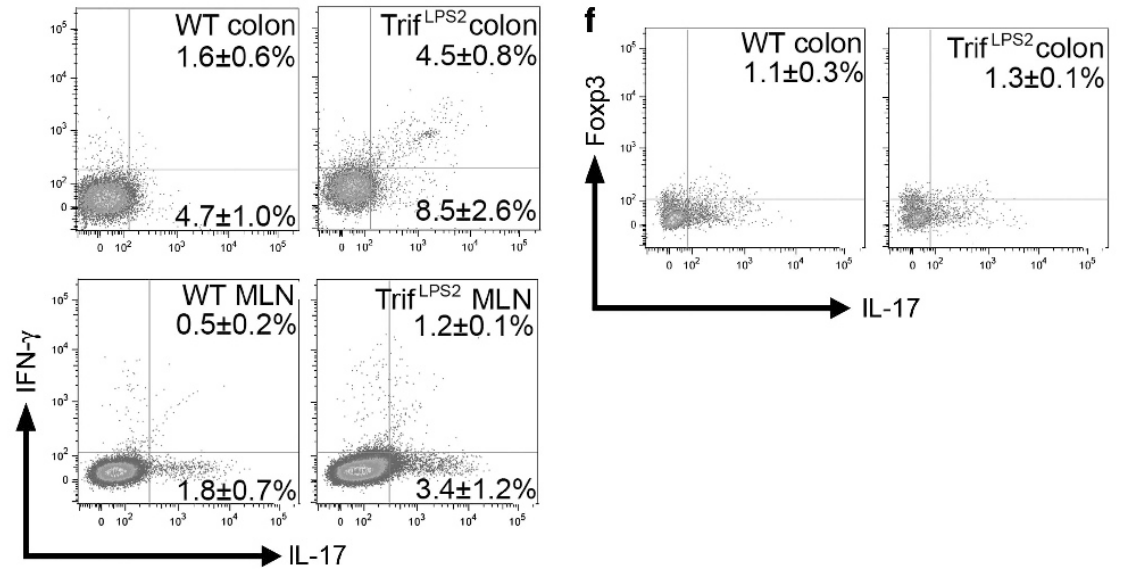

Figure 2 TRIF regulates interleukin (IL)-17-expressing CD4 ${ }^{+}$T cells in the intestine during 2,4,6-trinitrobenzenesulphonic acid (TNBS) colitis. (a) Realtime PCR analysis of the expression of IL-12p35, interferon (IFN)- $\gamma$, tumor necrosis factor (TNF- $\alpha$ ), and IL-17 in TNBS-treated wild-type (WT) and Trif ${ }^{\text {LPS2 }}$ mice $\left(n=9\right.$ each, ${ }^{\star} P<0.05$, NS, not significant). (b) Enzyme-linked immunosorbent assay analysis of IL-17 protein in ex vivo colon culture supernatants $\left(n=6\right.$ each, $\left.{ }^{*} P<0.05\right)$. (c) Flow cytometry (FCM) analysis of IFN- $\gamma$ and IL-17 in lamina propria CD4 ${ }^{+}$T cells after induction of TNBS colitis. Results are representative of two independent experiments demonstrating mean \pm s.e.m. ( $n=6$ each). (d) FCM analysis of IFN- $\gamma$ and IL-17 in CD4 ${ }^{+}$T cells isolated from the mesenteric lymph node (MLN) after induction of TNBS colitis. The representative results from two independent experiments are shown demonstrating mean \pm s.e.m. ( $n=6$ each). (e) FCM detection of IFN- $\gamma$-expressing Th17 cells in the intestine. CD4 ${ }^{+}$T cells isolated from the lamina propria and the MLN that express IFN- $\gamma$ and IL-17 are shown. The representative results from two independent experiments ( $n=6$ each, mean \pm s.e.m.). (f) FCM data showing Foxp3 and IL-17 expression in CD4 ${ }^{+}$T cells isolated from colonic lamina propria. The representative results from two independent experiments ( $n=6$ each, mean \pm s.e.m.). SSC, single-cell suspension. A full color version of this figure is available at the Mucosal Immunology journal online.

produced more IFN- $\beta$ protein than Trif ${ }^{\mathrm{LPS} 2}$ mice colon, there was no statistical difference between them. We also confirmed similar mucosal protein production of IL-6 and IL-12p70 between WT colon and Trif ${ }^{\mathrm{LPS} 2}$ colon (Figure $4 \mathbf{b}$ ). Immunohistochemistry was then used to examine which cell type in the colon expressed IL-27p28. Colonic samples from WT mice in TNBS colitis showed that most IL-27p28-immunopositive cells were positive for F4/80 but not for CD11c (Figure 4c). These results indicate that the expression of IL-27p28 in lamina propria macrophages is impaired in Trif ${ }^{\mathrm{LPS} 2}$ mice during TNBS colitis. Because IL-27p28 is known to suppress Th17-cell differentiation, ${ }^{21,22}$ defective mucosal production of IL-27p28 may be involved in abnormal generation of Th17 cells and their plasticity in Trif ${ }^{\mathrm{LPS} 2}$ mice in TNBS colitis. 

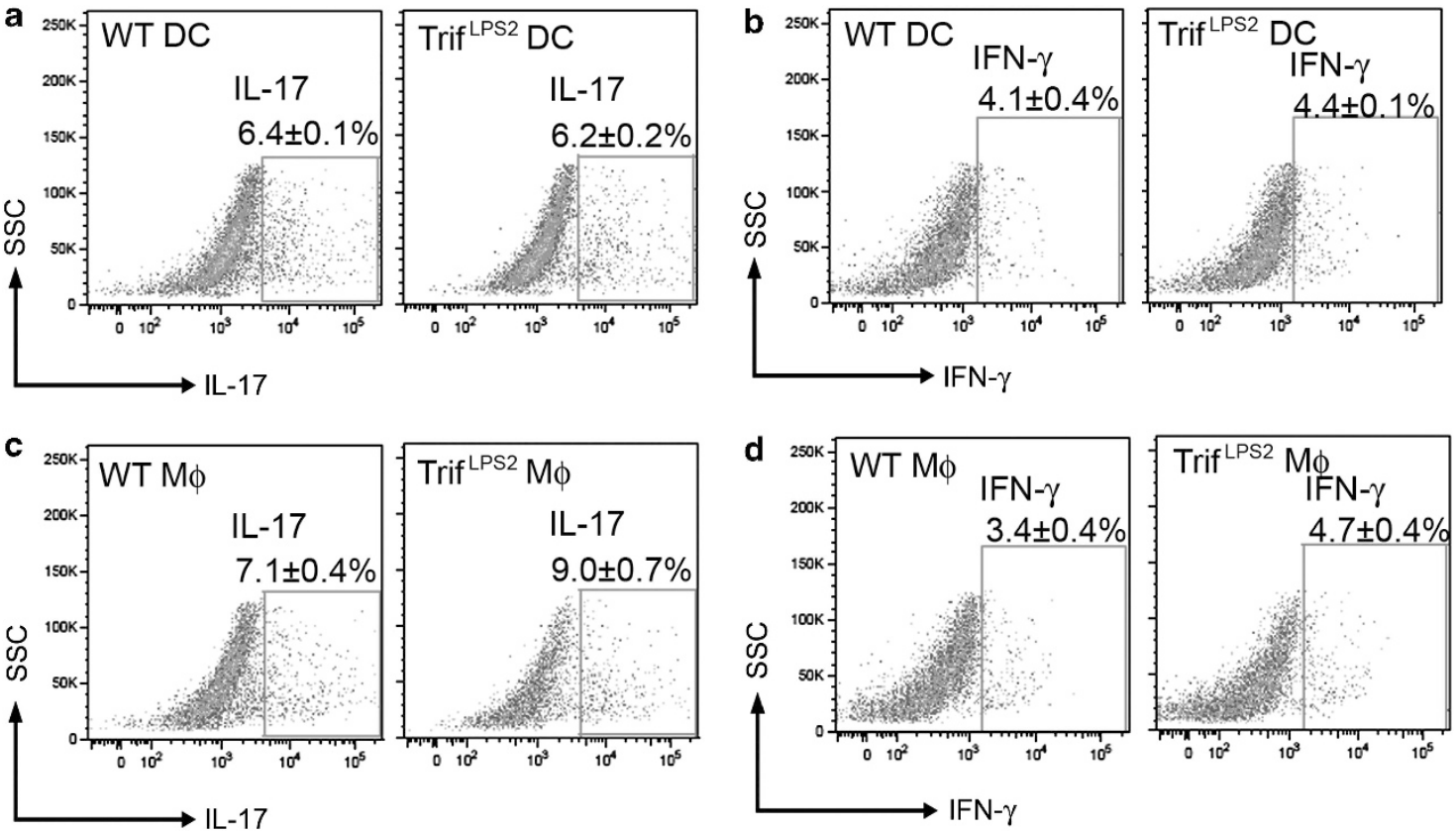

Figure 3 TRIF-deficient lamina propria dendritic cells (DCs) direct Th-cell differentiation to Th17 cells. Representative flow cytometry data of in vitro Th-cell differentiation. Wild-type (WT) naive T cells were differentiated with $\mathrm{F} 4 / 80^{-} \mathrm{CD}_{11 \mathrm{C}^{+}} \mathrm{LPDCs}$ or $\mathrm{F} 4 / 80^{+} \mathrm{CD} 11 \mathrm{c}^{-}$lamina propria macrophages from WT and Trif ${ }^{\text {LPS2 }}$ mice in the presence of cecal bacterial antigen. Intracellular cytokines interleukin (IL)-17 (a and $\mathbf{c}$ ) and interferon (IFN)- $\gamma$ (b and $\mathbf{d}$ ) in $\mathrm{CD} 4^{+}$T cells after 3 days are shown (cells were gated with $\mathrm{CD}^{+}$and $\mathrm{CD} 4^{+}$cells). The representative results from three independent experiments ( $n=4$ each, mean \pm s.e.m.). SSC, single-cell suspension. A full color version of this figure is available at the Mucosal Immunology journal online.

\section{TRIF-dependent expression of IL-27p28 by macrophages regulates IFN- $\gamma$-expressing Th17-cell generation in response to commensal bacteria}

Next, we examined whether the ability of Th17-cell generation differs between TRIF-deficient DCs and macrophages and that is associated with Th17/Th1 plasticity. WT naive T cells incubated with lamina propria CD11 $\mathrm{c}^{+}$cells (DCs) from WT mice in the presence of CBA did not generate Th17 cells that expressed IFN- $\gamma$ (Figure 5a). Even after co-culturing WT naive $\mathrm{T}$ cells with lamina propria CD11 ${ }^{+}$DCs from Trif ${ }^{\mathrm{LPS} 2}$ mice, cells that expressed both IL-17 and IFN- $\gamma$ were only barely detectable (Figure 5a). However, substantial generation of IFN- $\gamma$-expressing Th17 cells was observed with the use of Trif $^{\mathrm{LPS} 2}$ peritoneal macrophages instead of lamina propria $\mathrm{CD}_{11 \mathrm{c}^{+}}$DCs (Figure 5b).

Next, we examined the concentration of IFN- $\beta$ and IL-27p28 in the media taken from T-cell co-culture with lamina propria $\mathrm{CD}_{11 \mathrm{c}^{+}}$DCs or peritoneal macrophages. There was a striking difference in IL-27p28 concentrations between macrophage wells and DC wells (Figure 5c). In the supernatants of DC-Tcell co-cultures, the concentration of IL-27p28 was very low and there was no difference between the wells with WT DCs and Trif $^{\mathrm{LPS} 2}$ DCs. However, substantial IL-27p28 production was observed in supernatants of macrophage-T-cell co-cultures and the wells with WT macrophages showed significantly greater concentrations of IL-27p28 compared with the wells with Trif ${ }^{\mathrm{LPS} 2}$ macrophages. Because CBA contains multiple pathogen-associated molecular patterns, and TRIF receives both TLR3 and TLR4 inputs, we compared macrophage response with CBA, poly I:C, and lipopolysaccharide. Both poly $\mathrm{I}: \mathrm{C}$, and lipopolysaccharide induced IL-27p28 as much as CBA in $\mathrm{WT}$ peritoneal macrophages, but these responses were absent in Trif ${ }^{\mathrm{LPS} 2}$ peritoneal macrophages (Figure 5D). When we examined intracellular cytokine expression in T cells, naive $\mathrm{T}$ cells that were co-cultured with $\mathrm{Trif}^{\mathrm{LPS} 2}$ macrophages revealed a IFN- $\gamma$-expressing Th17-cell population. However, adding back IL-27p28 abolished abnormal generation of Th17 cells as well as IFN- $\gamma$ expression by Th17 cells during co-culturing with $\mathrm{Trif}^{\mathrm{LPS} 2}$ macrophages (Figure 5e). Therefore, TRIF-dependent IL-27p28 production might be a dominant factor that determines lineage commitment of Th1 cells from the intermediate state between Th17 cells during colitis.

\section{Exogenous supplementation of Trif ${ }^{\text {LPS2 }}$ mice with recombinant IL-27p28 reduced Th17 cells in the lamina propria and ameliorated severity of colitis}

In order to determine the physiological importance of IL-27p28 in intestinal generation and plasticity of Th17 cells in the context of intestinal inflammation, we treated Trif ${ }^{\mathrm{LPS} 2}$ mice with recombinant IL-27p28 during the course of TNBS colitis. The mice that received IL-27p28 preserved body weight compared to control Trif ${ }^{\mathrm{LPS} 2}$ mice (Figure 6a). Colonoscopy clearly showed improved mucosal appearance in IL-27p28 treated mice compared to control Trif ${ }^{\mathrm{LPS} 2}$ mice (Figure 6a). Histologically, control Trif ${ }^{\mathrm{LPS} 2}$ mice had multiple ulcerations with necrotic mucosa and total epithelial loss, while 
IL-27p28 treated Trif ${ }^{\text {LPS2 }}$ mice preserved epithelial cell lining even in the most damaged areas (Figure 6b). Furthermore, treatment with IL-27p28 not only reduced Th17 cells but also
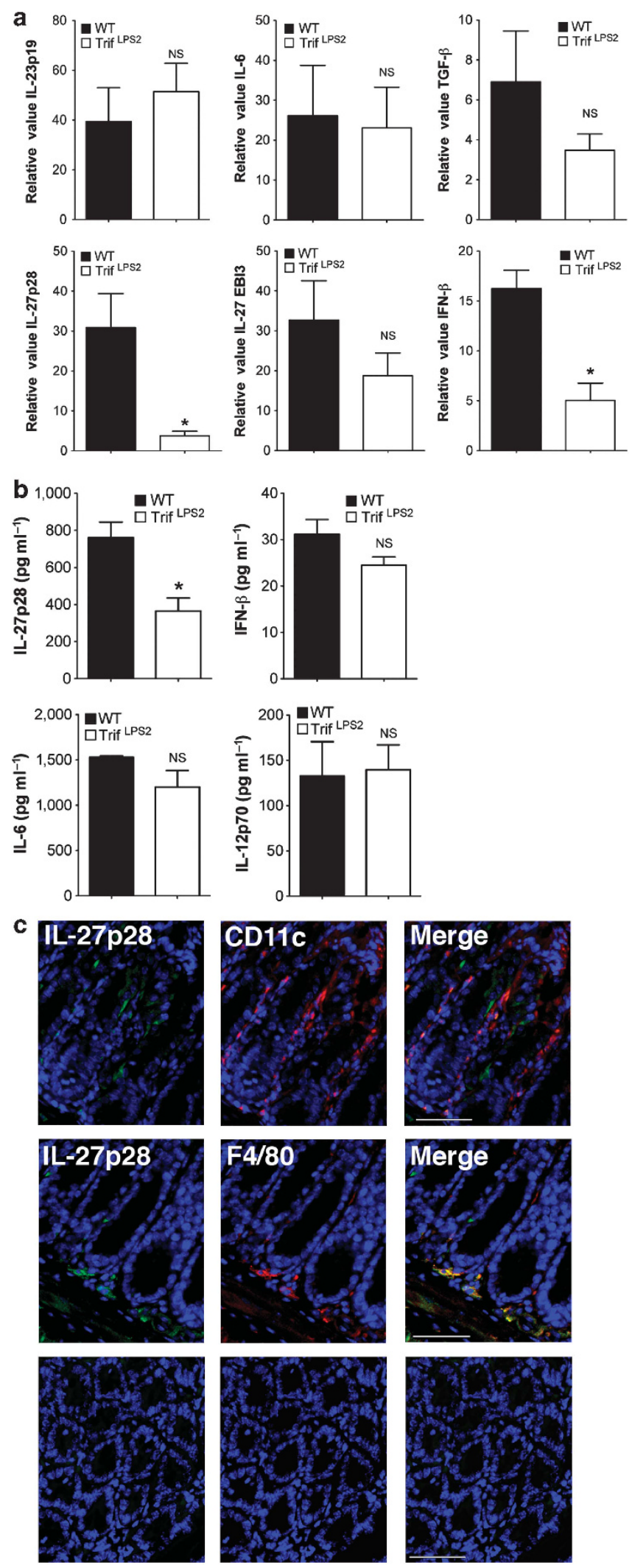

suppressed the generation of IFN- $\gamma$-expressing lamina propria Th17 cells (Figure 6c). These results indicate that IL-27p28 regulates abnormal generation of IFN- $\gamma$-expressing Th17 cells that are responsible for increased severity of colitis in Trif ${ }^{\text {LPS2 }}$ mice.

TRIF-dependent regulation of Th17-cell differentiation and plasticity is associated with impaired activation of T-cell STAT1

Given that IL-27p28 is the end effector in TRIF-dependent regulation of Th17/Th1-cell differentiation in the intestine, we sought to determine how IL-27p28 works on T cells during their differentiation. In order to determine the underlying mechanism, we examined the activation of transcriptional factors involved in Th17 and Th1 cell differentiation in our in vitro T-cell differentiation system. Naive $\mathrm{T}$ cells that were cocultured with Trif ${ }^{\mathrm{LPS} 2}$ macrophages in the presence of CBA demonstrated higher expression of ROR $\gamma \mathrm{t}$ than T cells with WT macrophages, which was consistent with the abundance of Th17 cells generated (Figures $\mathbf{7 a}$ and $\mathbf{5 d}$ ). The difference in STAT3 activation was subtle between $\mathrm{T}$ cells that were cocultured with Trif ${ }^{\text {LPS2 }}$ macrophages and $\mathrm{T}$ cells co-cultured with WT macrophages (Figure 7a). In contrast, STAT1 activation in $\mathrm{T}$ cells co-cultured with Trif ${ }^{\mathrm{LPS} 2}$ macrophages was strikingly lower compared with that in $\mathrm{T}$ cells co-cultured with WT macrophages (Figure 7a).

To determine the role of T-cell STAT1 activation in the regulation of Th17-cell differentiation and plasticity in the intestine, we used naive $\mathrm{T}$ cells isolated from STAT1 ${ }^{-/-}$ mice and incubated with WT or Trif ${ }^{\text {LPS2 }}$ macrophages in the presence of CBA. The majority of STAT $1^{-/-} \mathrm{T}$ cells were differentiated into Th17 cells in T cells co-cultured with WT macrophages that was more abundant than T cells with Trif ${ }^{\text {LPS2 }}$ macrophages (Figure $7 \mathbf{b}$ ). The IFN- $\gamma$-expressing population in Th17 cells was observed regardless of the genotype of macrophages used, suggesting that T-cell STAT1 activation strongly inhibits Th17-cell differentiation and may be indispensable for the regulation of Th17/Th1 plasticity (Figure $\mathbf{7 b}$ ). As STAT1 can be activated by IFN- $\beta$, which is induced through TRIF signaling, we next addressed whether blocking IFN- $\beta$ reduced STAT1 activation in T cells during differentiation. Results demonstrated that anti-IFNAR1 antibody reduced STAT1 activation and increased ROR $\gamma \mathrm{t}$ expression in T cells after co-culturing with WT macrophages and CBA (Figure 7c).

Figure $4 \quad$ Trif $^{L P S 2}$ mice had impaired expression of interleukin (IL)-27p28 in lamina propria macrophages during colitis. (a) Real-time PCR analysis of mucosal cytokine messenger RNA expression $\left(n=9\right.$ each, $\left.{ }^{*} P<0.05\right)$. (b) The protein production of IL-27p28, IL-6, and IL-12p70 from ex vivo colon culture supernatants determined by enzyme-linked immunosorbent assay $\left(n=6\right.$ each, $\left.{ }^{\star} P<0.05\right)$. (c) Representative immunofluorescent pictures of colonic mucosa (wild-type (WT) mice colon of in 2,4,6trinitrobenzenesulphonic acid (TNBS) colitis). Positive cells for IL-27p28 (green) and CD11c (red) or F4/80 (red) in the lamina propria are shown. Control slides (bottom panel) were processed without primary antibodies. Original magnification, $\times 20$, Bar $=50 \mu \mathrm{m}$. NS, not significant. 

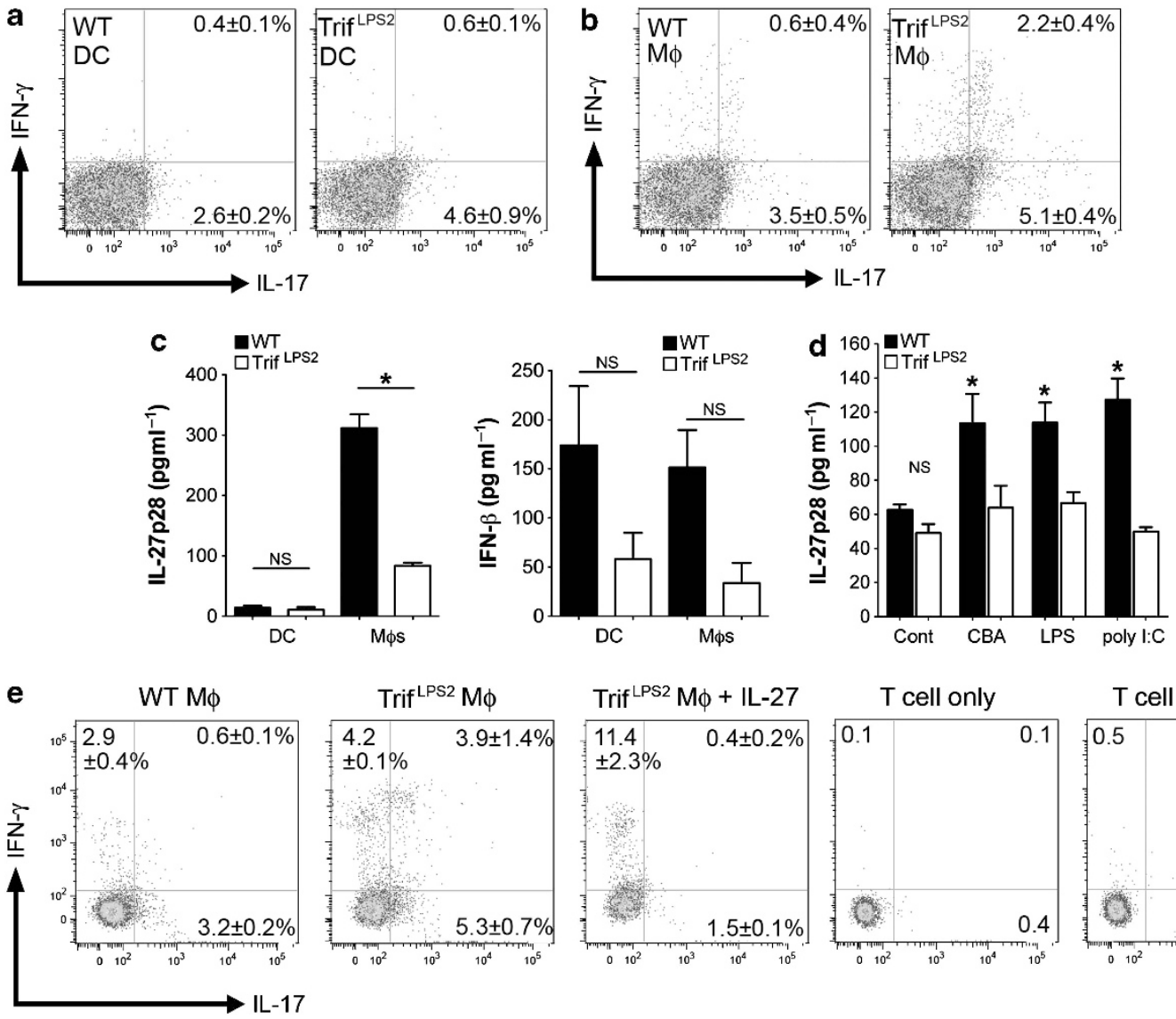

T cell + IL-27

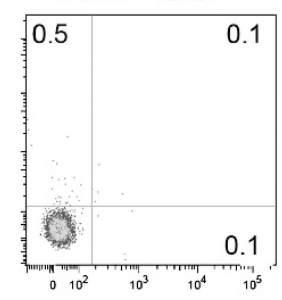

Figure 5 TRIF-dependent regulation of Th17-cell differentiation and plasticity is mediated through the induction of interleukin (IL)-27p28 in macrophages. (a) Expression of interferon (IFN)- $\gamma$ and IL-17 in CD4 ${ }^{+}$T cells after co-culturing with CD11c ${ }^{+}$lamina propria dendritic cells (DCs) in the presence of cecal bacterial antigen (CBA) for 3 days. The representative results from three independent experiments $(n=6$ each, mean \pm s.e.m.). (b) Expression of IFN- $\gamma$ and IL-17 in CD4 ${ }^{+}$T cells after co-culturing with peritoneal macrophages (MФs) in the presence of CBA for 3 days. Results are representative of three independent experiments ( $n=6$ each, mean \pm s.e.m.). (c) Enzyme-linked immunosorbent assay (ELISA) detection of IL-27p28 (left) and IFN- $\beta$ (right) protein concentration in the supernatants taken from co-cultures ( $n=6$ each, mean \pm s.e.m., ${ }^{*} P<0.05$, NS, not significant). (d) Macrophage response to toll-like receptor ligands. Peritoneal macrophages isolated from wild-type (WT) and Trif ${ }^{\text {LPS2 }}$ mice were stimulated with CBA $\left(100 \mu \mathrm{g} \mathrm{ml}^{-1}\right)$, poly I:C $\left(5 \mu \mathrm{g} \mathrm{ml}^{-1}\right)$, and lipopolysaccharide (LPS) $\left(500 \mathrm{ng} \mathrm{ml}^{-1}\right)$ for $24 \mathrm{~h}$. The supernatants were measured for concentration of IL-27p28 by ELISA. ( $n=3$ each, mean \pm s.e.m., ${ }^{*} P<0.05$, NS, not significant). (e) The effect of IL-27p28 on Th17-cell differentiation and plasticity induced by Trif $^{\mathrm{LPS} 2} \mathrm{M} \Phi$ s. Flow cytometry analysis of IL-17 and IFN- $\gamma$ in CD4 ${ }^{+}$T cells are shown after co-culturing with M $\Phi$ s in the presence or absence of IL-27p28 supplementation. Results are representative of two independent experiments ( $n=4$ each, mean \pm s.e.m.). A full color version of this figure is available at the Mucosal Immunology journal online.

Our results demonstrated that IL-27p28 reduced Th17-cell differentiation and generation of the IFN- $\gamma$-expressing Th17cell population in Trif ${ }^{\mathrm{LPS} 2}$ mice in vivo and in vitro (Figures 5d and $\mathbf{6 c}$ ). This effect of IL-27p28 on Th17-cell generation and Th17/Th1 plasticity is dependent on STAT1 activation because IL-27p28 did not reduce Th17-cell generation including IFN- $\gamma$-expressing population when we co-cultured Trif $^{\mathrm{LPS} 2}$ macrophages and STAT1 ${ }^{-1-}$ naive $\mathrm{T}$ cells in the presence of CBA (Figure 7d). Simple stimulation of WT splenocytes with IL-27p28 induced phosphorylation of STAT1 in $\mathrm{CD}^{+} \mathrm{T}$ cells (Figure 7e). These results suggest a model of regulatory mechanism by which Th17-cell generation is balanced with Th1-cell generation in the intestine.

\section{DISCUSSION}

Over a decade has passed since abnormal generation of Th17 cells was implicated in the pathogenesis of IBD. Despite extensive studies, it is still unclear how the abundant Th17 cells in intestinal mucosa contribute to chronic inflammation and how it is regulated during remission period. Using a mouse model of IBD, the current study demonstrates a specific contribution of TRIF signaling induced by commensal bacteria to the regulation of Th17-cell generation and plasticity during colitis. TRIF induced sequential expression of IFN- $\beta$ and IL-27p28 in macrophages in response to commensal bacteria that led to amplification of STAT1 activation in T cells. Timely activation of STAT1 prevents unstable plasticity of T cells and, therefore, uncontrolled production of IFN- $\gamma$ from Th17 cells. Our results highlight TRIF-mediated host-commensal interactions as an important regulator of Th17-cell generation and colitogenicity in the intestine.

Previous animal studies have demonstrated conflicting results regarding the role of Th17 cells in intestinal inflammation, demonstrating both colitogenic and regulatory properties 
a
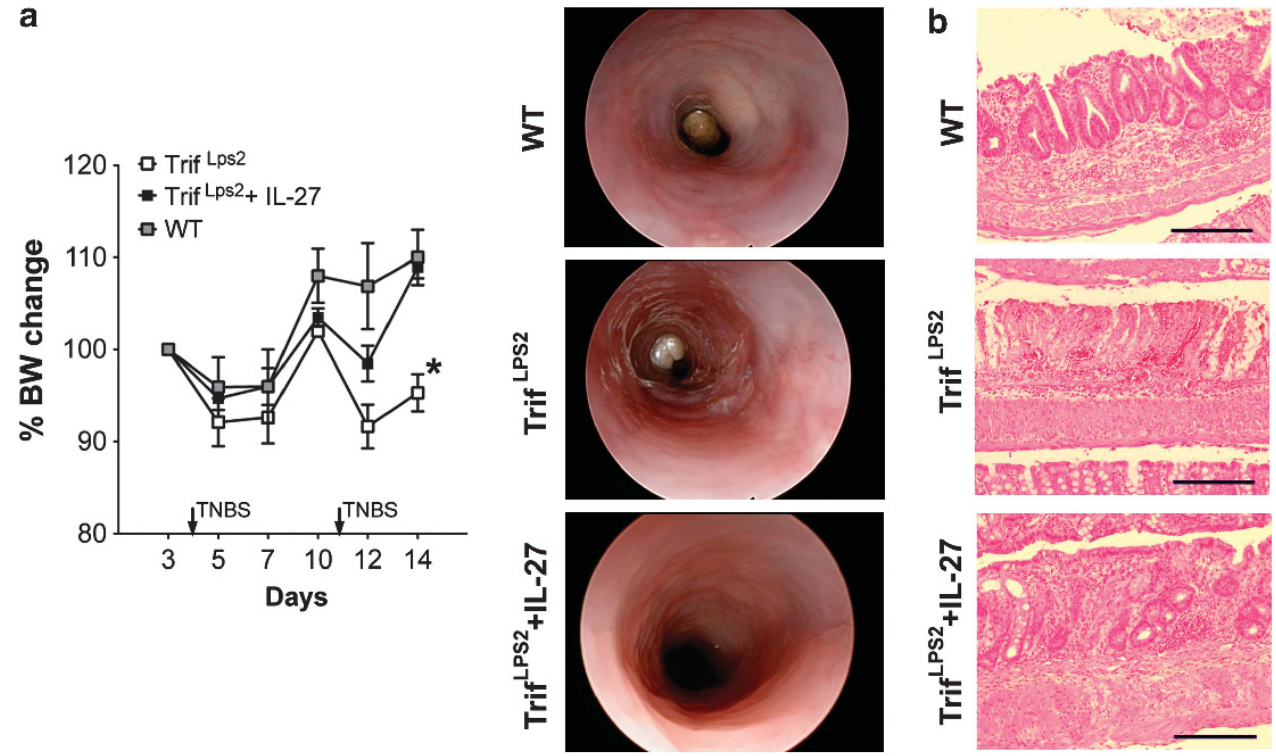

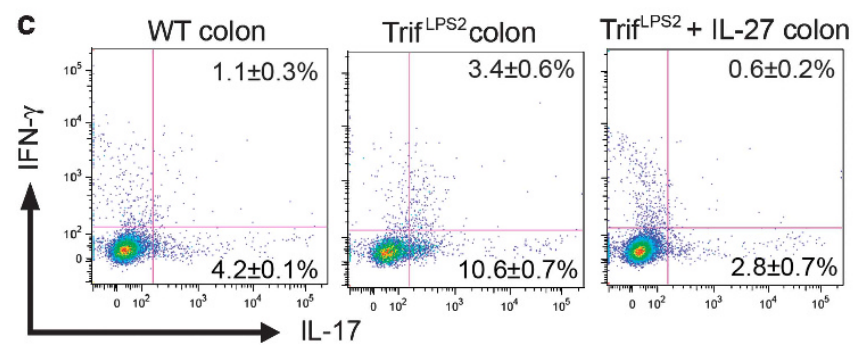

Figure 6 Interleukin (IL)-27p28 supplementation inhibits abnormal Th17-cell generation and intestinal pathology in Trif ${ }^{\text {LPS2 }}$ mice during colitis. Trif $^{\text {Lps2 }}$ mice were treated with recombinant IL-27p28 (intraperitoneal, daily $0.25 \mu$ g per mouse after 2,4,6-trinitrobenzenesulphonic acid (TNBS) injection) during the course of TNBS colitis. (a) The graph shows body weight change of the mice during TNBS colitis $(n=8$ in rIL-27p28-treated and non-treated Trif $^{\llcorner P S 2}$ mice, $n=5$ in wild-type (WT) mice, ${ }^{\star} P<0.05$ ). Combined data from two independent experiments are shown (mean \pm s.e.m.). Endoscopic findings in colonic mucosa (day 14 of TNBS colitis). (b) Representative photographs of hematoxylin and eosin staining of the colon taken from WT, rIL-27p28-treated and non-treated Trif ${ }^{\mathrm{LPS} 2}$ mice. Original magnification, $\times 20$, Bar $=100 \mu \mathrm{m}$. (c) Flow cytometry analysis of IL-17 and interferon (IFN)- $\gamma$ in lamina propria CD4 ${ }^{+}$T cells taken from WT, rlL-27p28-treated and non-treated Trif ${ }^{\mathrm{LPS} 2}$ mice (day 14 of TNBS colitis). The number shows mean \pm s.e.m. ( $n=4$ each). BW, body weight.

of Th17 cells in the setting of intestinal inflammation. ${ }^{15,20,23-26}$ These results suggest that Th17 cells may behave pathogenically as well as in a regulatory manner depending on upstream signaling in the intestine. In fact, the functional heterogeneity of Th17 cells appears to be involved in the pathogenesis of human and animal models of IBD. ${ }^{7,27}$ On the basis of the previous findings of mice deficient in IL-17 and IL-17R that are less susceptible to TNBS colitis, ${ }^{28,29}$ we used the TNBS colitis model to examine the colitogenic properties of Th17 cells. Although the other cell types expressing IL-17, i.e., innate lymphoid cells, may contribute to the induction of colitis, ${ }^{30}$ our results showed significant increase of lamina propria Th17 cells corresponding to the severity of colitis. Because Th17 cells may lose colitogenic capacity in the presence of Th1 cells, the balance generating these two cell types may be crucial for determining the outcome of the disease. ${ }^{31}$

Despite what is known about the mutually exclusive nature of Th cells, emerging evidence has suggested a dynamic plasticity of Th17 cells with Th1 as well as regulatory $\mathrm{T}$ cells. ${ }^{13,14,20,27}$ The existence of IFN- $\gamma$-expressing Th17 cells in the lamina propria and the MLN in Trif ${ }^{\mathrm{LPS} 2}$ mice, but not in WT mice during colitis, implies a role for TRIF signaling in Th17/Th1 cell plasticity. As Trif ${ }^{\text {LPS2 }}$ mice do not have Foxp3 + Th17 cells in the intestine, it is not likely that altered Th17-cell generation in Trif ${ }^{\text {LPS2 }}$ mice is due to the general instability of T-cell terminal differentiation. Although distinct cytokine patterns have a central role in Th-cell differentiation and their plasticity, ${ }^{32}$ Trif ${ }^{\mathrm{LPS} 2}$ mice did not have altered mucosal expression of major cytokines that are known to be involved in Th-cell differentiation. Instead, Trif ${ }^{\mathrm{LPS} 2}$ mice showed defective expression of IFN- $\beta$ and IL-27p28 messenger RNA in the intestine. IFN- $\beta$ has been shown to inhibit human Th17-cell differentiation by suppressing IL-23p19 expression and inducing IL-27p28 expression in DCs. ${ }^{22}$ In the intestine, macrophages seem to be the dominant source of IL-27p28 during colitis, as we demonstrated by immunochemistry. Trif $^{\text {LPS2 }}$ DCs and macrophages both enhanced Th17-cell differentiation in response to commensal bacteria, but IFN$\gamma$-expressing Th17 cells were only generated in the case of Trif $^{\mathrm{LPS} 2}$ macrophages. Macrophage expression of IL-27p28 
a

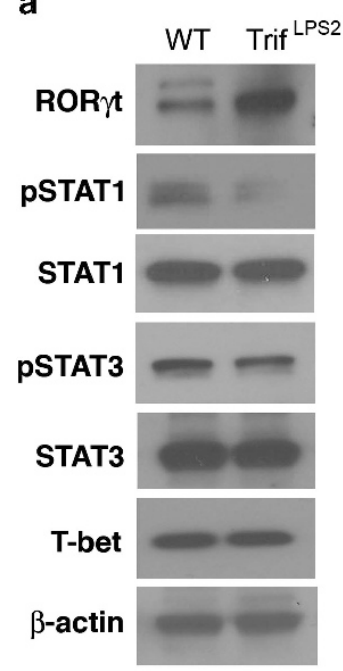

b

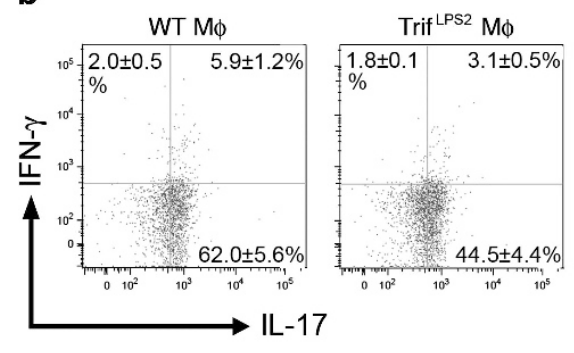

C

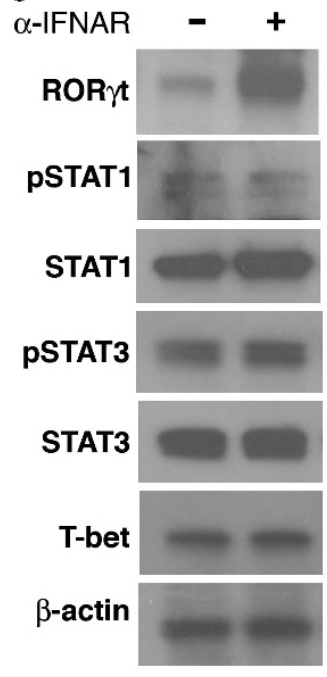

e $\quad 0 \mathrm{~min} \quad 30 \mathrm{~min} \quad 60 \mathrm{~min}$ d

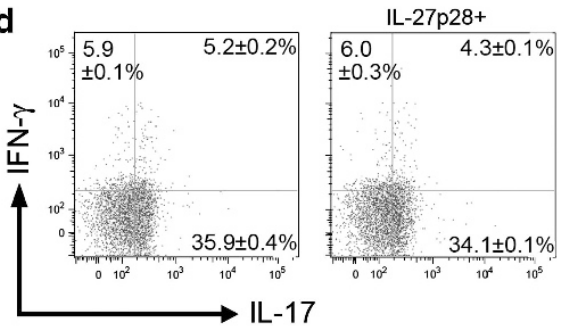

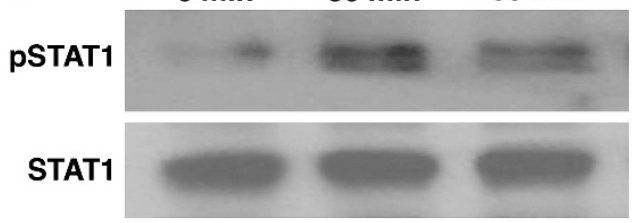

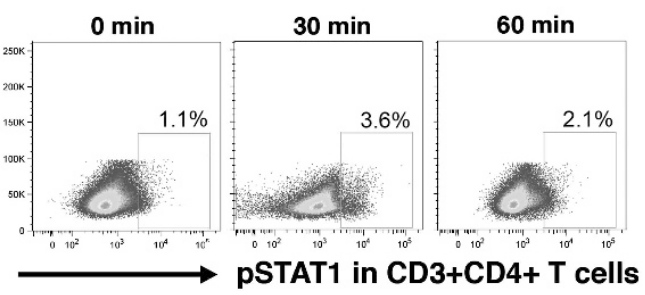

Figure 7 Interleukin (IL)-27p28-induced STAT1 activation regulates Th17-cell differentiation and plasticity. (a) Western blot analysis of transcription factors in wild-type (WT) naive T cells after co-culturing with MФs in the presence of cecal bacterial antigen (CBA). $\beta$-actin was used as a loading control. (b) Flow cytometry (FCM) analysis of IL-17 and interferon (IFN)- $\gamma$ in STAT ${ }^{-1-}$ naive T cells after in vitro differentiation with WT and Trif ${ }^{L P S} 2 \mathrm{M} \Phi$ in the presence of CBA. Representative results from five independent experiments are shown ( $n=4$ each, mean \pm s.e.m.). (c) Western blot analysis of transcription factors in WT naive T cells after co-culturing with WT MФs and CBA in the presence of anti-IFNAR1 antibody. (d) FCM analysis of IL-17 and IFN- $\gamma$ in STAT1 ${ }^{-1}-$ naive T cells after co-culturing with Trif ${ }^{\mathrm{LPS} 2} \mathrm{M} \Phi \mathrm{s}$ in the presence of CBA and recombinant IL-27p28 $\left(15 \mathrm{ng} \mathrm{ml}^{-1}\right)$. Representative results from two independent experiments are shown $(n=4$ each, mean \pm s.e.m.). (e) Western blot (top panel) and FCM (bottom panel) analysis of pSTAT1 in WT splenocytes after stimulation with IL-27p28 $\left(15 \mathrm{ng} \mathrm{ml}^{-1}\right)$ for the indicated period. For the FCM analysis, splenocytes were stained with CD3, CD4, and pSTAT1 after IL-27p28 stimulation. pSTAT1-positive cells in CD3 ${ }^{+}$CD4 ${ }^{+}$ splenocytes are demonstrated. Results are representative of two independent experiments $(n=4$ each, mean \pm s.e.m.). A full color version of this figure is available at the Mucosal Immunology journal online.

may be crucial for the regulation of Th17/Th1 plasticity because adding back IL-27p28 to Trif ${ }^{\mathrm{LPS} 2}$ mice restored the generation of Th17 cells to the level of the WT mice and suppressed IFN- $\gamma$ expression by Th17 cells. Although previous reports have suggested that IL-27p28 inhibits intestinal inflammation by inducing IL-10-expressing Tregs, this effect might be on other cell types than $\mathrm{T}$ cells. ${ }^{33-35}$ As there was no difference in number of lamina propria Treg cells between WT and Trif ${ }^{\mathrm{LPS} 2}$ mice during TNBS colitis, TRIF-dependent regulation of Th17cell differentiation and plasticity is unlikely to be mediated through Treg-cell generation in our model.

Accumulating evidence has suggested a significant role for STAT1 in the regulation of Th17-cell generation. ${ }^{22,36}$ Our results demonstrated that the absence of TRIF signaling in macrophages increased ROR $\gamma \mathrm{t}$ expression and decreased STAT1 activation in T cells in response to intestinal bacterial antigen, whereas T-cell STAT3 activation was not influenced by the presence or absence of TRIF signaling in macrophages. A similar activation pattern of transcription factors was observed when type I IFN signaling was blocked, suggesting T-cell STAT1 activation requires TRIF-dependent induction of IFN- $\beta$ in macrophages. Furthermore, abundant generation of IFN- $\gamma$ expressing Th17 cells was observed in STAT1 ${ }^{-1-} \mathrm{T}$ cells. IL-27p28 suppressed IFN- $\gamma$-expressing Th17-cell generation by Trif ${ }^{\mathrm{LPS} 2}$ macrophages but failed to suppress it in STAT1 ${ }^{-1-}$ $\mathrm{T}$ cells, indicating IL-27p28-mediated regulation of Th17/Th1 plasticity was STAT1 dependent. As STAT1 ${ }^{-1-}$ mice do not induce spontaneous inflammation in the intestine, this pathway 
may occur upon epithelial damage, where TRIF signaling is induced in response to commensal bacteria.

Clinical trials targeting Th17 cells have yielded discouraging results. ${ }^{4-6}$ Several gene mutations regarding human IL-27 have been associated with early-onset IBD, and IL-27 mutant patients with Crohn's disease have impaired expression of IL-27 in the colon. ${ }^{37}$ In this study, we showed a possible mechanism in abnormal generation of pathogenic Th17 cells that express IFN- $\gamma$ in the intestine. Further understanding of intestinal Th17-cell biology especially the regulatory mechanism of their plasticity may significantly contribute to improving the management of IBD patients.

\begin{abstract}
METHODS
Mice and induction of colitis. WT and Trif ${ }^{\mathrm{LPS} 2}$ mice were purchased from the Jackson Laboratory (Bar Harbor, ME). Six-week-old females were used in this study. Colitis was induced by rectal administration of $2.5 \mathrm{mg}$ TNBS (Sigma, St Louis, MO) dissolved in 55\% ethanol. Colitis was induced by sensitizing mice skin with $100 \mu \mathrm{l}$ of $2.5 \mathrm{mg}$ TNBS in $55 \%$ ethanol followed by rectal administration of TNBS in $55 \%$ ethanol $(2.5 \mathrm{mg}$ in $200 \mu \mathrm{l})$ on days 4 and 11 . To ensure distribution of the TNBS in the colon, mice were held in a vertical position for $1 \mathrm{~min}$ post injection. Control mice were treated with $55 \%$ ethanol alone. Body weight was measured every other day and calculated as percent change in weight compared with baseline. Mice were euthanized on day 14 , the colon removed, washed out luminal contents with phosphate-buffered saline, and weight and length measured to calculate weight/length ratio as described previously. ${ }^{38}$ The colon tissue was fixed in $10 \%$ buffered formalin, paraffin embedded, sectioned, and stained with hematoxylin and eosin. Histological assessment was performed by a veterinary pathologist masked to the mouse genotype and treatment. Histologic score was a combined score of crypt damage (0-4), acute inflammatory cell infiltrate (0-4), edema (0-4), erosion/ulceration (0-4), chronic inflammatory cell infiltrate $(0-2)$, epithelial regeneration $(0-3)$, and crypt distortion/branching (0-3).
\end{abstract}

Mouse colonoscopy. Mucosal appearance of TNBS colitis was determined by colonoscopy by using an Olympus Exera CV-160 camera system (Olympus, Tokyo, Japan).

Cell preparation and purification. Single-cell suspensions from the spleen and the MLNs were prepared by mechanical disruption with $70 \mu \mathrm{m}$ nylon mesh. The colon was removed and flushed with Hank's balanced salt solution containing $5 \%$ fetal bovine serum. The colon was cut into small pieces and shaken at 200 r.p.m. for 20 min at $37^{\circ} \mathrm{C}$ with $\mathrm{Ca}^{++} \mathrm{Mg}^{++}$free Hank's balanced salt solution containing $5 \%$ fetal bovine serum and $0.5 \mathrm{~m}$ EDTA. The colon pieces were further shaken at 200 r.p.m. for $60 \mathrm{~min}$ at $37^{\circ} \mathrm{C}$ with RPMI1640 containing $5 \%$ fetal bovine serum, collagenase VIII $\left(30 \mathrm{U} \mathrm{ml}^{-1}\right)$ (Sigma) and trypsin inhibitor $\left(0.24 \mathrm{mg} \mathrm{ml}^{-1}\right)$ (Sigma). Lamina propria cells were collected by filtering through a $70-\mu \mathrm{m}$ cell strainer and then purified with lymphocyte-separation medium (Cellgro, Corning, NY) by centrifugation at $800 \mathrm{~g}$ for $20 \mathrm{~min}$ at $20^{\circ} \mathrm{C}$.

Preparation of CBA. CBA was prepared using C57Bl6 mice as previously described with minor modifications. ${ }^{39}$ In brief, the cecums were removed from $\mathrm{C} 57 \mathrm{Bl} 6$ mice and placed in $1 \mathrm{ml}$ of phosphatebuffered saline containing $0.05 \%$ Tween 20 . After addition of $2.5 \mathrm{~mm}$ protease inhibitor and $2.5 \mathrm{mg} \mathrm{ml}^{-1} \mathrm{SDS}$, the suspension was heated at $56^{\circ} \mathrm{C}$ for $30 \mathrm{~min}$. The solid components were disrupted using stainless steel beads (Qiagen, Valencia, CA) using Tissue lyser II (Qiagen) for $3 \mathrm{~min}$ at $30 \mathrm{~Hz}$. The cecal lysate was passed through a 0.2 -micron syringe filter. Protein concentration was measured using the Bradford protein assay kit (Bio-Rad, Hercules, CA).
Real-time PCR. Total RNA was isolated from tissue samples using RNA Bee (Tel-Test, Friendwood, TX) according to the manufacturer's instructions. A total of $1 \mu \mathrm{g}$ RNA was used as the template for singlestrand complementary DNA synthesis using the QuantiTect Reverse Transcription Kit (Qiagen). Quantitative real-time PCR was performed for IL-12p35, IFN- $\beta$, tumor necrosis factor- $\alpha$, IL-17, IL-23p19, IL-6, TGF- $\beta$, IL-27p28, IL-27EBI3, IFN- $\gamma$, and $\beta$-actin (Supplementary Table 1). The complementary DNA was amplified using Maxima SYBR Green/ROX (Thermo scientific, Pittsburgh, PA) on the Lightcycler (Roche, Indianapolis, IN). The amplification results of the genes of interest were normalized to the corresponding $\beta$-actin results and analyzed using the delta-delta method. Data were expressed as fold induction relative to the lowest one.

FCM. Surface staining of CD69, CD3, and CD4 was performed according to the manufacturer's instructions (eBioscience, San Diego, CA). Intracellular staining of IFN- $\gamma$ and IL-17 was performed after stimulation with PMA $\left(50 \mathrm{ng} \mathrm{ml}^{-1}\right)$, ionomycin $\left(350 \mathrm{ng} \mathrm{ml}^{-1}\right)$, and GogiPlug $\left(0.8 \mu \mathrm{ml}^{-1}\right)$ for $4 \mathrm{~h}$. FCM analyses were performed on an LSRII flow cytometer with FACS Diva (Becton Dickenson, Franklin Lakes, NJ) and FlowJo software (Tree Star, Ashland, OR).

In vitro T-cell differentiation. Lamina propria macrophages were purified from lamina propria mononuclear cells using anti-F4/80-PE and anti-PE microbeads (Miltenyi Biotec, Auburn, CA). After elution of anti-F4/80-PE and anti-PE microbeads, CD11c + cells were excluded by using $\mathrm{CD} 11_{\mathrm{C}}$ (N418) microbeads (Miltenyi Biotec). On the other hand, LPDCs were purified from lamina propria mononuclear cells after exclusion of F4/80 + cells. Peritoneal macrophages were isolated from peritoneal lavage as described previously. ${ }^{40} \mathrm{WT}$ naive $T$ cells from splenocytes were purified by magnetic sorting using the CD4 $+\mathrm{T}$ cells Isolation Kit II (Miltenyi Biotec) with CD62L (L-selection) microbeads (Miltenyi Biotec). WT naive T cells were cocultured with DCs or macrophages (5:1 ratio) with CBA $\left(100 \mu \mathrm{g} \mathrm{ml}^{-1}\right)$ for $72 \mathrm{~h}$. T cells were analyzed for IFN- $\gamma$ and IL-17 by FCM after gating $\mathrm{CD} 3+\mathrm{CD} 4+$ cells. Anti-IFNAR1 antibody $\left(15 \mu \mathrm{g} \mathrm{ml}^{-1}\right.$ ) (Leinco Technologies, St Louis, MO) was used to block type I IFN signaling. After $72 \mathrm{~h}$ of co-culture, differentiated T cells were also subjected to western blot analysis.

Enzyme-linked immunosorbent assay. Protein levels of IFN- $\beta$, IL-27p28, and IL-6 were determined in the cell/tissue culture supernatants by using the DuoSet ELISA Development System (R\&D Systems, Minneapolis, MN) according to the manufacturer's instructions. For ex vivo colonic tissue cultures, $100 \mathrm{mg}$ of colon tissue was incubated in $1 \mathrm{ml}$ serum-free RPMI1640 for $24 \mathrm{~h}$.

Western blot analysis. Lysate $(25 \mu \mathrm{g})$ was subjected to $10 \%$ SDSpolyacrylamide gel electrophoresis and transferred to Immun-Blot PVDF membranes (Bio-Rad). After blocking with 5\% milk containing $2 \%$ bovine serum albumin, the membrane was blotted with antibodies against ROR $\gamma$ t (ab78007), T-bet (ab91109), pSTAT1 (ab29045), STAT1 (ab92509) which were purchased from Abcam (Cambridge, MA), and pSTAT3 (9145S), STAT3 (9132), and $\beta$-actin (4967S) from Cell Signaling Technology (Beverly, MA), followed by suitable secondary antibody conjugated with horseradish peroxidase. The membrane was exposed on radiographical film using the SuperSignal West Dura kit (Thermo Scientific).

Immunofluorescent staining. Freshly isolated colon samples were fixed in $10 \%$ neutral buffered formalin and embedded in paraffin. Four-micrometer sections were incubated in 5\% skim milk for $1 \mathrm{~h}$ and stained with rat anti-mouse IL-27p28 antibody (1:200) overnight at $4 \mathrm{C}$ followed by Alexa fluor 488 anti-rabbit IgG (1:200) (Invitrogen, Carlsbad, CA) for $90 \mathrm{~min}$ at room temperature. Sections were then re-incubated with $5 \%$ skim milk for $1 \mathrm{~h}$ followed by staining with antimouse CD11c (1:100, Invitrogen) or anti-mouse F4/80 (1:100, Serotec) immunuglobulin Gs. Alexa fluor 568-conjugated rabbit anti-rat IgG (1:200) was used as secondary antibody. Sections were counterstained 
with 4',6-diamidino-2-phenylindole, dihydrochloride and staining specificity was confirmed by omitting primary antibodies. Stained slides were examined using an EVOS fluorescent microscope (Advanced Microscopy Group, Bothell, WA).

Statistical analysis. Student's $t$-test for the comparison of two independent samples and one-way analysis of variance for $>2$ independent samples were performed using GraphPad Prism (Version $5.0 \mathrm{~b}$, San Diego, CA), and a $P$-value of $<0.05$ was considered statistically significant.

SUPPLEMENTARY MATERIAL is linked to the online version of the paper at http://www.nature.com/mi

\section{ACKNOWLEDGMENTS}

Part of this study was supported by the NIH grant Al095255 and a Senior Research Award from Crohn's and Colitis Foundation of America.

\section{DISCLOSURE}

The authors declared no conflict of interest.

(c) 2015 Society for Mucosal Immunology

\section{REFERENCES}

1. Round, J.L. \& Mazmanian, S.K. The gut microbiota shapes intestinal immune responses during health and disease. Nat. Rev. Immunol. 9, 313323 (2009).

2. McGovern, D. \& Powrie, F. The IL23 axis plays a key role in the pathogenesis of IBD. Gut 56, 1333-1336 (2007).

3. Becker, C. et al. Constitutive $\mathrm{p} 40$ promoter activation and IL-23 production in the terminal ileum mediated by dendritic cells. J. Clin. Invest. 112, 693706 (2003).

4. Mannon, P.J. et al. Anti-interleukin-12 antibody for active Crohn's disease. N. Engl. J. Med. 351, 2069-2079 (2004).

5. Sandborn, W.J. et al. A randomized trial of Ustekinumab, a human interleukin-12/23 monoclonal antibody, in patients with moderate-tosevere Crohn's disease. Gastroenterology 135, 1130-1141 (2008).

6. Hueber, W. etal. Secukinumab, a human anti-IL-17A monoclonal antibody, for moderate to severe Crohn's disease: unexpected results of a randomised, double-blind placebo-controlled trial. Gut 61, 1693-1700 (2012).

7. Symons, A., Budelsky, A.L. \& Towne, J.E. Are Th17 cells in the gut pathogenic or protective?. Mucosal Immunol. 5, 4-6 (2012).

8. O'Connor, W. Jr., Zenewicz, L.A. \& Flavell, R.A. The dual nature of $T(H) 17$ cells: shifting the focus to function. Nat. Immunol. 11, 471-476 (2010).

9. Mucida, D. T-helping colitis. Gastroenterology 141, 801-805 (2011).

10. McGeachy, M.J. et al. TGF-beta and IL-6 drive the production of IL-17 and IL-10 by $\mathrm{T}$ cells and restrain $\mathrm{T}(\mathrm{H})-17$ cell-mediated pathology. Nat. Immunol. 8, 1390-1397 (2007).

11. Louten, J., Boniface, K. \& de Waal Malefyt, R. Development and function of TH17 cells in health and disease. J. Allergy Clin. Immunol. 123, 1004-1011 (2009).

12. Bi, Y., Liu, G. \& Yang, R. Reciprocal modulation between $\mathrm{TH} 17$ and other helper T cell lineages. J. Cell. Physiol. 226, 8-13 (2011).

13. Hovhannisyan, Z., Treatman, J., Littman, D.R. \& Mayer, L. Characterization of interleukin-17-producing regulatory Tcells in inflamed intestinal mucosa from patients with inflammatory bowel diseases. Gastroenterology 140 , 957-965 (2011).

14. Sarra, M., Pallone, F., Macdonald, T.T. \& Monteleone, G. IL-23/L-17 axis in IBD. Inflamm. Bowel Dis. 16, 1808-1813 (2010).

15. Atarashi, K. et al. ATP drives lamina propria $\mathrm{T}(\mathrm{H}) 17$ cell differentiation. Nature 455, 808-812 (2008).

16. Ivanov, I.I. et al. Specific microbiota direct the differentiation of IL-17producing T-helper cells in the mucosa of the small intestine. Cell Host Microbe 4, 337-349 (2008).

17. Shaw, M.H., Kamada, N., Kim, Y.G. \& Nunez, G. Microbiota-induced IL-1beta, but not IL-6, is critical for the development of steady-state TH17 cells in the intestine. J. Exp. Med. 209, 251-258 (2012).
18. Guo, B., Chang, E.Y. \& Cheng, G. The type I IFN induction pathway constrains Th17-mediated autoimmune inflammation in mice. J. Clin. Invest. 118, 1680-1690 (2008).

19. De Luca, A. et al. Functional yet balanced reactivity to Candida albicans requires TRIF, MyD88, and IDO-dependent inhibition of Rorc. J. Immunol. 179, 5999-6008 (2007).

20. Ahern, P.P. et al. Interleukin-23 drives intestinal inflammation through direct activity on T cells. Immunity 33, 279-288 (2010).

21. Diveu, C. et al. IL-27 blocks RORc expression to inhibit lineage commitment of Th17 cells. J. Immunol. 182, 5748-5756 (2009).

22. Ramgolam, V.S., Sha, Y., Jin, J., Zhang, X. \& Markovic-Plese, S. IFN-beta inhibits human Th17 cell differentiation. J. Immunol. 183, 5418-5427 (2009).

23. Kullberg, M.C. et al. IL-23 plays a key role in Helicobacter hepaticusinduced T cell-dependent colitis. J. Exp. Med. 203, 2485-2494 (2006).

24. Elson, C.O. et al. Monoclonal anti-interleukin 23 reverses active colitis in a T cell-mediated model in mice. Gastroenterology 132, 2359-2370 (2007).

25. O'Connor, W. Jr. et al. A protective function for interleukin 17A in T cellmediated intestinal inflammation. Nat. Immunol. 10, 603-609 (2009).

26. Leppkes, M. et al. RORgamma-expressing Th17 cells induce murine chronic intestinal inflammation via redundant effects of IL-17A and IL-17F. Gastroenterology 136, 257-267 (2009).

27. Lee, Y.K. et al. Late developmental plasticity in the Thelper 17 lineage. Immunity 30, 92-107 (2009).

28. Zhang, Z., Zheng, M., Bindas, J., Schwarzenberger, P. \& Kolls, J.K. Critical role of IL-17 receptor signaling in acute TNBS-induced colitis. Inflamm. Bowel Dis. 12, 382-388 (2006).

29. Jin, Y., Lin, Y., Lin, L. \& Zheng, C. IL-17/IFN-gamma interactions regulate intestinal inflammation in TNBS-induced acute colitis. J. Interferon Cytokine Res. 32, 548-556 (2012).

30. Geremia, A. et al. IL-23-responsive innate lymphoid cells are increased in inflammatory bowel disease. J. Exp. Med. 208, 1127-1133 (2011).

31. Mikami, Y. et al. Competition between colitogenic Th1 and Th17 cells contributes to the amelioration of colitis. Eur. J. Immunol. 40, 2409-2422 (2010).

32. Basu, R., Hatton, R.D. \& Weaver, C.T. The Th17 family: flexibility follows function. Immunol. Rev. 252, 89-103 (2013).

33. Hanson, M.L. et al. Oral delivery of IL-27 recombinant bacteria attenuates immune colitis in mice. Gastroenterology 146, 210-21, e13 (2014).

34. Visperas, A., Do, J.S., Bulek, K., Li, X. \& Min, B. IL-27, targeting antigenpresenting cells, promotes Th17 differentiation and colitis in mice. Mucosal Immunol. 7, 625-633 (2013).

35. Mascanfroni, I.D. et al. IL-27 acts on DCs to suppress the Tcell response and autoimmunity by inducing expression of the immunoregulatory molecule CD39. Nat. Immunol. 14, 1054-1063 (2013).

36. Villarino, A.V., Gallo, E. \& Abbas, A.K. STAT1-activating cytokines limit Th17 responses through both T-bet-dependent and-independent mechanisms. J. Immunol. 185, 6461-6471 (2010).

37. Imielinski, M. et al. Common variants at five new loci associated with earlyonset inflammatory bowel disease. Nat. Genet. 41, 1335-1340 (2009).

38. Kjellev, S., Lundsgaard, D., Poulsen, S.S. \& Markholst, H. Reconstitution of Scid mice with CD4 + CD25- T cells leads to rapid colitis: an improved model for pharmacologic testing. Int. Immunopharmacol. 6, 1341-1354 (2006).

39. Cong, $Y$. et al. CD4 + T cells reactive to enteric bacterial antigens in spontaneously colitic $\mathrm{C} 3 \mathrm{H} / \mathrm{HeJBir}$ mice: increased $\mathrm{T}$ helper cell type 1 response and ability to transfer disease. J. Exp. Med. 187, 855-864 (1998).

40. Sotolongo, J. et al. Host innate recognition of an intestinal bacterial pathogen induces TRIF-dependent protective immunity. J. Exp. Med. 208, 2705-2716 (2011).

41. Fukata, M. et al. The myeloid differentiation factor 88 (MyD88) is required for $\mathrm{CD} 4+\mathrm{T}$ cell effector function in a murine model of inflammatory bowel disease. J. Immunol. 180, 1886-1894 (2008).

This work is licensed under the Creative Commons Attribution-NonCommercial-No Derivative Works 3.0 Unported License. To view a copy of this license, visit http://creativecommons.org/licenses/by-nc-nd/3.0/ 